\section{Climate change predicted to increase kidney stone prevalence in the US}

Global warming will increase the incidence of nephrolithiasis and the costs of its treatment, suggests a modeling study by Brikowski et al. The US geographical area at high risk is expected to increase with a northward and westward expansion of the 'kidney stone belt'. Similar changes are likely to occur in Europe and Asia, which will increase the global burden of kidney stone disease.

Geographical and seasonal variations in the risk of stone formation support a potential association between increased temperature and raised kidney stone prevalence, possibly attributable to dehydration and resultant decreased urine volume. For this study, the authors used two mathematical models derived from previous data and the climate-change predictions of the intermediate-warming scenarios defined by the Intergovernmental Panel on Climate Change to explore future changes in prevalence of stone disease. The two models were respectively based on linear or nonlinear relationships between temperature and the risk of stone formation. The linear model predicted an increase in stone prevalence of $10.4 \%$ by 2050, whereas the nonlinear model predicted an increase of $7.0 \%$. Treatment costs were predicted to rise by $25 \%$ over this period. Use of a severe climate change scenario resulted in greater increases in prevalence (15-20\%) and cost (up to $40 \%$ ).

The authors note that true stone prevalence is difficult to determine, as many patients with kidney stones do not seek treatment. The researchers recommend further study using national data sets that include self-reported cases.

Original article Brikowski TH et al. (2008) Climate-related increase in the prevalence of urolithiasis in the United States. Proc Natl Acad Sci USA 105: 9841-9846

\section{Novel therapies for renal cell carcinoma: studies in mouse models}

The poor prognosis associated with metastatic renal cell carcinoma (RCC) and the modest clinical efficacy of many existing treatments has prompted research into novel therapies for this disease. Two recent studies in mouse models illustrate some of the promising treatment approaches for this malignancy.

Doehn et al. had previously shown that an adjuvant, autologous, renal tumor cell vaccine (Reniale ${ }^{\circledR}$, LipoNova GmbH, Hannover, Germany) reduced the risk of tumor progression in humans after radical nephrectomy. Now, in a study published in European Urology, these authors have investigated the efficacy and mode of action of an analogous vaccine, derived from a murine RCC cell line, in a mouse model. After five doses of vaccine, female $B A L B / c$ mice received an injection of viable tumor cells into the kidney, followed by a final dose of vaccine. Tumor growth and metastasis was significantly impaired in these animals compared with an unvaccinated control group. With regard to mode of action, adoptive immune transfer experiments indicated a cellbased antitumoral effect, mainly attributable to the activation and maintenance at elevated levels of a population of tumor-specific CD8 ${ }^{+}$ $T$ lymphocytes. The authors conclude that the vaccine might work in the clinical setting by preventing progression of micrometastases after resection of the primary tumor. The researchers suggest that their results offer insight into which outcome variables are suitable for use in clinical trials to elucidate the mode of action of such vaccines in humans.

In a separate trial, Mellon et al. investigated antiangiogenic gene therapy in a mouse model of RCC. Bilateral flank tumors were induced in athymic (nude) mice via the injection of a human RCC cell line. Subsequent unilateral injection of an adenovirus vector engineered to deliver DNA that encoded a chimeric human endostatin-angiostatin protein with antiangiogenic properties resulted in significant suppression of both ipsilateral and contralateral local tumor growth (>90\% inhibition at 50 days) in vector-treated animals compared with control animals that received only saline or an alternative adenovirus $(P<0.001)$. In vivo dual-photon microscopy demonstrated poorly developed vasculature in the endostatinangiostatin-treated mice, and serum assays confirmed a systemic effect. Compared with control mice, treated mice had significantly better 60 -day survival $(100 \%$ vs $0 \%, P<0.001)$ and freedom from lung or liver metastases on postmortem tissue analysis ( $100 \%$ vs $<22 \%$, $P<0.001)$. The authors conclude that their 